\title{
Hereditary glaucoma
}

INSERM

\section{Source}

INSERM. (1999). Orphanet: an online rare disease and orphan drug data base. Hereditary glaucoma. ORPHA:359

Hereditary glaucoma is a clinically diverse group of rare eye disorders with genetic predisposition characterized by elevated intraocular pressure (IOP) and glaucomatous changes of the optic nerve head, leading to field defects, visual loss and blindness. Hereditary glaucoma can be sub-classified as primary (congenital glaucoma, juvenile glaucoma) or secondary according to the presence or absence of systemic or other ocular anomalies (iridogoniodysg enesis, Stickler syndrome, Coats syndrome). The clinical presentation is variable and is based on age, severity of glaucoma, presence of ocular abnormalities and development of secondary IOP related abnormalities. 